\title{
Las redes sociales como salida profesional para los estudiantes de comunicación de España y América Latina
}

\section{Social networks as a career opportunity for Communication students in Spain and Latin America}

\author{
Santiago Tejedor*, Santiago Giraldo Luque**, Ricardo Carniel Bugs*** \\ * Universitat Autònoma de Barcelona, España \\ ** Universitat Autònoma de Barcelona, España \\ *** Universitat Autònoma de Barcelona, España
}

Resumen

\begin{abstract}
El estudio de las redes sociales entre los jóvenes internautas (generación "milennial") se ha convertido en una línea de investigación que ha generado numerosos trabajos y artículos. Sin embargo, apenas se ha estudiado la visión de estas plataformas como escenarios de desarrollo profesional de los futuros comunicadores. Este artículo presenta los resultados de una investigación realizada en 16 países con 1.080 estudiantes de comunicación que reflexionaron sobre la posibilidad de trabajar a futuro en una red social. El estudio, que reunió un total de 32.400 evidencias, concluye que los estudiantes de comunicación están presentes en las redes sociales y valoran positivamente su uso, pero que no han tomado conciencia de las posibilidades que, a nivel profesional, éstas les ofrecen.
\end{abstract}

Palabas clave: Redes sociales, Comunicación, Milennials, Jóvenes, Empleo.

Abstract

The analysis of social networks among young Internet users ("Millennial" generation) has become a research field that has generated numerous studies and articles. However, the visualisation of these platforms as scenarios of professional careers development of the future communicators has scarcely been studied. This article presents the results of a research carried out in 16 countries with 1,080 students of Communication who thought about the possibility of working in a social, digital network. The study, which gathered a total of 32,400 evidences, concludes that communication students are present in social networks and value their use positively, but they have not become aware of the professional possibilities that they offer them.

Keywords: Social networks, Communication, Milennials, Youth, Employment.

\section{La "Generación Y" y sus hábitos en el consumo de medios}

La generación "milennial", "Generación del Milenio" o "Generación Y" (como evolución terminológica de la denominada "Generación $X$ " que les precede) se ha convertido en objeto de estudio desde diferentes disciplinas (Francese, 2003). En el ámbito de la comunicación, la importancia de esta franja generacional se proyecta en dos grandes escenarios. Por un lado, su rol como consumidores activos o "prosumidores" (Toffler, 1980) de los contenidos generados en el ciberespacio y, por otro, su capacidad para liderar una serie de transformaciones en el uso de las plataformas digitales centradas en la generación y distribución de contenidos. Aunque no existe un consenso exacto respecto al año de inicio de la misma, la mayoría de estudios la sitúa en un rango de edad que comprende los nacidos entre 1980 y 1994 (McCrindle \& Wolfinger, 2010).

Diferentes investigaciones han identificado cambios en el tipo de consumo de los contenidos digitales a partir de franjas de edades. En el informe sobre el estado de las noticias en los medios "State of the News 
Media 2014: Overview' (Pew Research, 2014), se identificaba, entre otros asuntos, la existencia de una brecha generacional en el consumo de noticias a través de canales interactivos y de la televisión. La investigación indicaba que cerca de la mitad de los usuarios adultos de internet en Estados Unidos consultaron información on-line sobre política básicamente a partir de Facebook. El estudio puntualizaba que los usuarios de Facebook (62\%) utilizan esta red para acceder a información política en mayor medida que los usuarios de Twitter (40\%) u otras redes sociales. El porcentaje de usuarios de la denominada "Generación $Y$ " que utilizaron Facebook para buscar recursos digitales sobre temas políticos (61\%) contrastaba ampliamente con la de los llamados "Baby Boomers" (de edades entre 50 y 67 años), que, en su mayoría, preferían informarse mediante la televisión (60\%). Asimismo, el informe apuntaba una serie de transformaciones en el consumo de los mensajes informativos on-line. Según la citada investigación, el $50 \%$ de los usuarios de redes sociales estadounidenses compartía o republicaba noticias, imágenes o vídeos, y aportaba sus comentarios a las noticias en estas plataformas dialógicas. Un año después, en 2015, el organismo regulador del audiovisual británico señalaba, respecto al consumo televisivo de los jóvenes, que la franja de entre 16 y 34 años que se informó a través de la televisión bajó un 29\% de 2008 a 2014 (Ofcom, 2015).

Un año más tarde, un informe conjunto del Pew Research Center y la Knight Foundation (Pew Research Center, 2016) identificaba un claro aumento del número de ciudadanos que se informan a través de Twitter y Facebook. Más de la mitad de los usuarios de Twitter (63\%) y Facebook (63\%) consideran estas dos plataformas sociales como una fuente para obtener información fuera del círculo de los amigos y las familias. Este porcentaje aumentó considerablemente desde 2013 cuando era menor el número de usuarios de Twitter (52\%) y de Facebook (47\%) que manifestaban informarse a través de estas dos redes sociales. Poco tiempo después, un estudio del American Press Institute (2016) indicaba que la búsqueda de información era la principal motivación para acceder a Facebook de un $47 \%$ de los jóvenes.

América Latina, por su parte, posee uno de los mayores índices de utilización de las redes sociales del mundo, según establece un estudio de la CEPAL (2015) que señalaba que el $78,4 \%$ de los usuarios de internet latinoamericanos participaban en las redes sociales. Este porcentaje superaba incluso al de América del Norte $(64,6 \%)$ y al de Europa (54,5\%), que poseen, no obstante, una mayor penetración de la tecnología en sus respectivos países. Entre las redes sociales más utilizadas en los países latinoamericanos, se encuentran, por este orden: Facebook, LinkedIn, Twitter y Taringa (red social de origen argentino que se utiliza ampliamente en los países de habla hispana y en la comunidad latina afincada en Estados Unidos). En este contexto latinoamericano, donde el número de estudios diagnósticos sobre la Generación Y es más reducido, destaca el informe "Telefonica Global Millennial Survey" (Telefónica, 2014) que señalaba que el $78 \%$ de los jóvenes latinoamericanos de entre 18 y 30 años, disponen de un smartphone. Según el estudio, los usuarios utilizaban estos aparatos, principalmente y según este orden, para: acceder a las redes sociales, leer mensajes y hacer llamadas. La encuesta, que interrogaba a los jóvenes sobre sus hábitos y costumbres tecnológicas, estableció que un elevado porcentaje de ellos (71\%) estarían dispuestos a viajar al extranjero en busca del trabajo ideal, un porcentaje que incluso era superior en el contexto latinoamericano (81\%). En el escenario español, existen diversas investigaciones sobre el uso profesional que los alumnos del ámbito de la comunicación realizan de plataformas como Facebook, Twitter o similares. Una investigación de la Universidad de Murcia (Martínez \& Torrado, 2011) analizó el uso de las redes sociales como fuente de información en sus estudiantes de periodismo y estableció que la mayoría poseía perfiles 
en redes sociales $(87 \%)$, pero solo un grupo reducido (10\%) las usaban para consultar noticias u otros contenidos informativos digitales.

En este escenario, la investigación "Uso de las redes sociales por los estudiantes de comunicación de América Latina y España", de la que se derivan los resultados que se exponen en los siguientes apartados del artículo, estudió la concepción que los jóvenes estudiantes de comunicación poseen de las redes sociales como salida profesional. El estudio, que se desarrolló en 16 países, contó con la colaboración de 20 universidades y reunió un total de 32.400 evidencias a partir de la consulta a 1.080 estudiantes. El artículo analiza, a partir de la concepción y usos de las redes sociales de los encuestados, si los alumnos valoran estas plataformas como escenarios donde desarrollarse profesionalmente o si, por el contrario, las identifican únicamente con plataformas dialógicas on-line destinadas a la búsqueda, el intercambio de información o al ocio y al entretenimiento.

\section{Redes sociales y empleo}

Tuñez, (2010), Harrison y Barthel (2009) o Allan y Thorsen (2009) han destacado la importancia de las redes sociales en las rutinas de producción de los contenidos informativos, así como en los procesos de distribución y consumo de los mismos. Estas plataformas se han consolidado como una fuente básica en el consumo de noticias, al convertir los mensajes informativos en unidades "portátiles", "personalizadas" y "participativas" (Matsa \& Mitchell, 2014). Se trata de un conjunto de transformaciones que afectan igualmente a la concepción que los jóvenes usuarios de la red poseen de las diferentes herramientas y plataformas colaborativas del ciberespacio, así como del tipo de uso que hacen de las redes sociales (Waycott et al, 2010). No obstante, un aspecto de interés es conocer por qué los usuarios crean su perfil en estas plataformas. En este sentido, diferentes autores han ahondado en las motivaciones (Baek, Holton, Harp, \& Yaschur, 2011) o justificaciones (Whiting \& Williams, 2013) que llevarían a los internautas a decidirse a utilizar una red social. Bulut y Doğan (2017) han llegado incluso a proponer una clasificación de los usos de las redes sociales a partir de siete razones específicas de los usuarios. Entre ellas, la última motivación identificada alude al uso de las redes sociales con una finalidad empresarial o laboral. Dentro de esta motivación es posible diferenciar entre el uso de las redes para promocionar un trabajo específico (Baek, Holton, Harp, \& Yaschur, 2011) o para publicar avances profesionales y adquirir estatus social (Papacharissi \& Mendelson, 2011). Este tipo de usos abarcan a usuarios de todo tipo (Ruano, Congote \& Torres, 2016) sin que importe el tipo de relación o experiencia inicial que tengan con la tecnología: nativo tecnológico, inmigrante tecnológico, reflexivo tecnológico o escéptico tecnológico.

Sin embargo, no se clarifica si las redes sociales son concebidas únicamente como una vía de promoción de mensajes o si además los usuarios las perciben como posibles escenarios de desarrollo profesional. En el caso de los estudiantes de comunicación, debido a la particular coyuntura profesional del sector en el que deben desenvolverse, las redes sociales juegan un papel decisivo como salida profesional que, en tal caso, demandaría de una formación específica en tales herramientas. A ello se une que el crecimiento del uso de las redes sociales con fines profesionales se ha producido paralelamente a la irrupción de nuevos roles dentro de los medios digitales que demandan, entre otras, amplias habilidades en el manejo avanzado de estas plataformas digitales. 
En este sentido, el estudio "Perfiles profesionales de la industria de contenidos digitales en España 20122017" alude a las oportunidades profesionales que los proyectos digitales han inaugurado (FTI-Ametic, 2012). En la misma línea, desde 2014, se ha detectado un incremento de la oferta de puestos de trabajo vinculados con iniciativas on-line del ámbito comunicativo (Pew Research Center, 2014). Dicho estudio establecía que, en ese año, se crearon 5.000 puestos de trabajo en los medios de comunicación digitales estadounidenses. En el escenario iberoamericano, una reciente investigación realizada por los portales «Universia» y «Trabajando.com» (Diario Gestión, 2015) establecía que el 37\% de los jóvenes consideraban las redes sociales como una de las vías idóneas para promocionar sus contenidos y entablar contacto con su público. El estudio, que encuestó a 7.319 jóvenes de Argentina, Brasil, Chile, Colombia, México, Perú, Puerto Rico, España y Portugal, concluyó que los jóvenes consideran prioritario para las empresas tener una presencia destacada de las plataformas virtuales, entre ellas, las redes sociales.

En el marco de nuestro estudio, se planteó el reto de esclarecer si los estudiantes de comunicación consideran las redes sociales como una opción profesional. Para ello, resulta decisivo conocer cómo conciben estas plataformas y, del mismo modo, qué rol les otorgan en sus aspiraciones y expectativas profesionales.

\section{Material y métodos}

El estudio constituye la última fase de un proyecto de investigación en el que han participado universidades de 16 países de Iberoamérica. Las universidades, tanto públicas como privadas, han sido seleccionadas a partir de una revisión de los principales rankings académicos (como el THE WUR 2016-2017, el "QS World University", el ranking elaborado por el grupo SCIMAGO y directorio elaborado por Quacquarelli Symonds). La combinación de universidades públicas (6) y privadas (14) se ha realizado intencionadamente para acceder de forma más completa al perfil de destinatarios que persigue el estudio: estudiantes de comunicación, principalmente de primer y segundo curso. La investigación tiene como principal objetivo realizar un sondeo sobre cómo los estudiantes de comunicación de América Latina y España conciben, valoran y utilizan las redes sociales en los procesos de acceso, consulta y producción de contenidos digitales on-line. A partir de estos resultados, se buscaba determinar la importancia que le confieren a las mismas como herramientas de inserción profesional.

El estudio se basa en una encuesta realizada en al menos una universidad de cada uno de los 16 países de la muestra, tal y como se describe en la tabla 1.

\begin{tabular}{|l|l|l|}
\hline \multicolumn{2}{|c|}{ Tabla 1: Muestra de investigación } \\
\hline País & Universidades analizadas & Carácter \\
\hline \multirow{3}{*}{ Argentina } & Universidad Austral de Buenos Aires & Privada \\
\hline \multirow{3}{*}{ Chile } & Faculdade Cásper Líbero (Sao Paulo) & Privada \\
\cline { 2 - 3 } & Universidade Federal de Alagoas (Maceió) & Pública \\
\cline { 2 - 3 } & Universidade de Santa Cruz do Sul & Privada \\
\hline Colombia & Universidad Católica del Norte de Antofagasta & Privada \\
\hline Costa Rica & Universidad Pontificia Bolivariana (Medellín) & Privada \\
\hline Cuba & Universidad Nacional & Pública \\
\hline Ecuador & Universidad de La Habana & Pública \\
\hline \multirow{2}{*}{ El Salvador } & Universidad San Francisco de Quito & Privada \\
\hline
\end{tabular}




\begin{tabular}{|l|l|l|}
\hline España & Universidad Autónoma de Barcelona & Pública \\
\hline Guatemala & Universidad Mariano Gálvez (Ciudad de Guatemala) & Privada \\
\hline \multirow{2}{*}{ Honduras } & Universidad Nacional Autónoma de Honduras (Tegucigalpa) & Pública \\
\cline { 2 - 3 } & Universidad de San Pedro de Sula & Privada \\
\hline México & Universidad Autónoma de Nuevo León & Pública \\
\hline Nicaragua & Universidad Centroamericana de Managua (Managua) & Privada \\
\hline Paraguay & Universidad Autónoma de Asunción & Privada \\
\hline Perú & Universidad de Lima & Privada \\
\hline $\begin{array}{l}\text { República } \\
\text { Dominicana }\end{array}$ & Pontificia Universidad Católica Madre y Maestra (Santo Domingo) & Privada \\
\hline Elaboración propia & \\
\hline
\end{tabular}

La encuesta obtuvo un total de 1080 respuestas, con un promedio de 76 respuestas por país. A partir de la información disponible en las universidades participantes en el estudio, se encontró una media de 983 estudiantes de comunicación como población en cada universidad. La encuesta logra así alcanzar al 5,5\% del universo del estudio. En la encuesta participaron estudiantes universitarios de entre 15 y 24 años, siendo los 19 años la moda de la muestra (30,87\%) y los 20,3 años la media de edad. Los datos encontrados en las universidades estudiadas revelan que los estudiantes de periodismo o comunicación en los centros educativos de la muestra son principalmente mujeres (60\%). La encuesta refleja también esa proporción. Así, la mayoría de las encuestas fueron contestadas por mujeres (64\%), mientras que el 36\% fueron hombres. La mayoría de los estudiantes encuestados se ubican entre el primer (26\%) y segundo año de estudios (34,6\%). Las encuestas fueron aplicadas entre noviembre de 2015 y junio de 2016.

La metodología de trabajo se basa en una encuesta matriz de 30 preguntas que cruzaba preguntas de tipo cuantitativo con cuestiones abiertas que han permitido un trabajo más cualitativo en la lectura e interpretación de las respuestas de los alumnos. A través de los cuestionarios cerrados se han analizado, entre otras, las siguientes cuestiones: a) el nivel de conocimiento de las redes sociales; b) el tipo de uso y la importancia que los estudiantes de comunicación les otorgan en los procesos de detección, selección, consulta y producción de contenidos informativos multimedia; c) el seguimiento y uso que hacen de redes sociales profesionales (de periodistas o medios de comunicación, por ejemplo); d) la presenciad de redes sociales profesionales en el ecosistema digital de los estudiantes de comunicación y, finalmente, d) su concepción de las redes sociales como posibles escenarios de desarrollo profesional. Los datos cuantitativos se han analizado a partir de frecuencias y de porcentajes. Las variables principales de las preguntas de los cuestionarios han sido las siguientes: a) Concepción de una red social (en su desempeño profesional y en su tiempo de ocio); b) Valoración de las opciones profesionales que, a su juicio, ofrecen las redes sociales; c) Reflexión sobre los hábitos de uso de las redes sociales; d) Exposición de las posibles sinergias entre las redes sociales y los medios de comunicación.

Las preguntas abiertas, por su parte, se han centrado en conocer los motivos del uso de las redes sociales y, entre otros asuntos, solicitar a los encuestados propuestas de definición o reflexiones sobre el uso de estas herramientas digitales. La propuesta metodológica incluye preguntas cerradas dicotómicas para los temas más definidos sobre el uso, la valoración de las plataformas y el impacto de las redes sociales en desempeño cotidiano y profesional. Además, se ha considerado pertinente incorporar preguntas categorizadas (ofreciendo al encuestado una serie de listados de posibles actividades a desarrollar en una red social o diferentes nombres de plataformas dialógicas on-line) y, de este modo, se han combinado preguntas de identificación, de intención, de información y de opinión. 
Las preguntas de la encuesta que hacen parte del presente artículo forman parte de una investigación longitudinal acerca de la percepción de los estudiantes de comunicación de América Latina y España sobre las redes sociales, en el que participan la Universitat Autònoma de Barcelona y la Universidad Pontificia Bolivariana, de Medellín (Colombia). Resultados comparados de la investigación han sido ya publicados en diferentes estudios (Pérez Tornero et al, 2015; Tejedor et al, 2018). El cuestionario fue validado por un panel de expertos $(n=8)$ antes de ser implementado. Posteriormente, se realizó una versión electrónica de la encuesta que fue enviada a profesores de las universidades recogidas en la tabla 1. El cuestionario fue así difundido y aplicado por profesores de las universidades de la muestra a sus estudiantes universitarios quienes respondieron en formato online a las preguntas del cuestionario.

\section{Análisis y resultados}

La investigación se centró, en una primera etapa, en preguntar a los estudiantes en qué medio de comunicación les gustaría desarrollarse como profesionales del ámbito comunicativo. El objetivo de este primer interrogante era el de definir un primer escenario de acción que permitiera establecer sus preferencias a nivel profesional. Los encuestados debían puntuar del 1 al 6 (siendo 1 la opción menos valorada). Resulta llamativo que la opción "Internet" (21,46\%) ocupe el tercer puesto en número de respuestas como la opción más deseada. La red de redes supera a las opciones de "Prensa" y "Radio" que, aunque a poca distancia, se sitúan como las menos valoradas por los futuros comunicadores. Este aspecto se ve reforzado por los porcentajes que obtiene Internet en los niveles de 4 y 5 , convirtiéndose en la salida profesional más demandada por los estudiantes de acuerdo a los niveles de puntaje (con un $24,24 \%$ y un $22,39 \%$, respectivamente), superando incluso a la televisión. De este modo, resulta posible afirmar que los estudiantes de comunicación de América Latina y España contemplan Internet, a nivel general, como un escenario donde conseguir empleo y, además, se encuentra entre sus principales preferencias.

Tabla 2: ¿En qué medio te gustaría desarrollarte como profesional?

\begin{tabular}{|c|c|c|c|c|c|c|}
\hline & Prensa & Radio & Televisión & Fotografía & Internet & Otros \\
\hline $\mathbf{1}$ & $11,2 \%$ & $9,3 \%$ & $6,2 \%$ & $7,7 \%$ & $4,1 \%$ & $14,8 \%$ \\
\hline $\mathbf{2}$ & $12,4 \%$ & 11,3 & $8,7 \%$ & $9,7 \%$ & $6,2 \%$ & $6,7 \%$ \\
\hline $\mathbf{3}$ & $13,6 \%$ & $13,5 \%$ & $12,4 \%$ & $13,2 \%$ & $11,5 \%$ & $12,6 \%$ \\
\hline $\mathbf{4}$ & $16,5 \%$ & $15,5 \%$ & $14,5 \%$ & $18,5 \%$ & $22,3 \%$ & $11,9 \%$ \\
\hline $\mathbf{5}$ & $15,7 \%$ & $21,7 \%$ & $19,8 \%$ & $18,0 \%$ & $24,2 \%$ & $11,6 \%$ \\
\hline $\mathbf{6}$ & $21 \%$ & $20,1 \%$ & $32,6 \%$ & $23,1 \%$ & $21,5 \%$ & $19,6 \%$ \\
\hline $\mathbf{N} / \mathbf{C}$ & $9,3 \%$ & $8,2 \%$ & $5,5 \%$ & $9,5 \%$ & $9,9 \%$ & $22,5 \%$ \\
\hline
\end{tabular}

Fuente: Elaboración propia.

Las preferencias identificadas de acuerdo al carácter de universidades (públicas y privadas) guardan patrones similares. Al comparar las preferencias de los estudiantes sobre el número de respuestas para las universidades públicas y privadas existe una diferencia de menos de 5 puntos porcentuales en 32 casos de 36 posibles. Las diferencias destacables se presentan en una preferencia de 5 puntos positivos en la máxima valoración (6) que otorgan los estudiantes de las universidades públicas por trabajar en Internet, así como 
una diferencia de 6,5 puntos positivos también de las universidades públicas sobre las privadas de trabajar en prensa escrita en la quinta valoración (5). El comparativo puede apreciarse en la tabla 3.

Tabla 3: Preferencias por tipo de universidad (pública o privada)*

\begin{tabular}{|c|c|c|c|c|c|c|c|c|c|c|c|c|c|c|c|c|c|c|}
\hline & \multicolumn{3}{|c|}{ Prensa } & \multicolumn{3}{|c|}{ Radio } & \multicolumn{3}{|c|}{ TV } & \multicolumn{3}{|c|}{ Fotografía } & \multicolumn{3}{|c|}{ Internet } & \multicolumn{3}{|c|}{ Otros } \\
\hline & Pri & Púb & Dif & Pri & Púb & Dif & Pri & Púb & Dif & Pri & Púb & Dif & Pri & Púb & Dif & Pri & Púb & Dif \\
\hline 1 & 12,9 & 9,1 & 3,8 & 9,9 & 8,6 & 1,2 & 6,7 & 5,8 & 0,9 & 7,6 & 8,0 & 0,4 & 4,9 & 3,1 & 1,8 & 11,4 & 19,5 & 8,1 \\
\hline 2 & 12,2 & 12,6 & 0,4 & 11,4 & 11,3 & 0,1 & 9,1 & 8,2 & 0,9 & 8,7 & 11,1 & 2,3 & 7,2 & 5,1 & 2,1 & 5,1 & 9,1 & 4,0 \\
\hline 3 & 15,3 & 11,5 & 3,7 & 14 & 12,9 & 1,1 & 12,6 & 12 & 0,6 & 12,9 & 13,7 & 0,9 & 11,3 & 12 & 0,7 & 13,0 & 12,2 & 0,8 \\
\hline 4 & 17,2 & 15,5 & 1,6 & 16,2 & 14,6 & 1,6 & 16,2 & 12,2 & 4,0 & 17,6 & 20,0 & 2,3 & 22,7 & 21,7 & 1,0 & 12,4 & 11,3 & 1,1 \\
\hline 5 & 13 & 19,5 & 6,5 & 21,3 & 22,4 & 1,1 & 18,6 & 21,7 & 3,1 & 19,4 & 16,2 & 3,2 & 24,6 & 23,7 & 0,9 & 13,0 & 9,5 & 3,5 \\
\hline 6 & 19,7 & 22,8 & 3,1 & 18,4 & 22,6 & 4,2 & 30,8 & 35,3 & 4,4 & 25,0 & 20,4 & 4,6 & 19,4 & 24,4 & 5,0 & 23,7 & 14,0 & 9,7 \\
\hline$N / C$ & 9,7 & 8,9 & 0,8 & 8,7 & 7,5 & 1,2 & 6,0 & 4,9 & 1,1 & 8,7 & 10,6 & 1,9 & 9,9 & 10,0 & 0,1 & 21,3 & 24,4 & 3,1 \\
\hline
\end{tabular}

Fuente: Elaboración propia.

En términos de género, tampoco se aprecian diferencias importantes en las preferencias de los estudiantes. Las puntuaciones sobre su interés de trabajar en Internet tan sólo varían 1,1 puntos en promedio para los 6 niveles de preferencia. Las únicas diferencias mayores a cinco puntos (4 en total), se identifican en radio ( 2 casos) y en fotografía (1 caso). Los hombres prefieren la radio $(24,9 \%)$ con la máxima puntuación (6) frente a las mujeres (17,3\%). En el caso inverso, las mujeres señalan a la radio con su valoración más baja (1) en un 11,3\%, mientras que los hombres lo hacen en un 5,5\%. En el caso de la fotografía, el 19,9\% de las mujeres otorgan la puntuación de 5, mientras que el 17,7\% de los hombres le otorga esta puntuación. El último caso evidenciado hace referencia o "otros".

El panorama comparativo entre los países de América Latina (850 respuestas) y España (220 respuestas) refleja, sin embargo, algunas distancias en las preferencias, sobre todo en la prensa, la radio, y alguna valoración de Internet. En estos medios las diferencias pueden alcanzar incluso los 16 puntos. Sin embargo, las tendencias de máximos y mínimos sobre su prevalencia en apreciar a Internet como un futuro profesional es bastante similar, como se aprecia en la Tabla 4.

Tabla 4: Preferencias por ámbito geográfico (pública o privada)

\begin{tabular}{|c|c|c|c|c|c|c|c|c|c|c|c|c|c|c|c|c|c|c|}
\hline & \multicolumn{3}{|c|}{ Prensa } & \multicolumn{3}{|c|}{ Radio } & \multicolumn{3}{|c|}{ TV } & \multicolumn{3}{|c|}{ Fotografía } & \multicolumn{3}{|c|}{ Internet } & \multicolumn{3}{|c|}{ Otros } \\
\hline & $\mathrm{AL}$ & ESP & Dif & $\mathrm{AL}$ & ESP & Dif & $\mathrm{AL}$ & ESP & Dif & $\mathrm{AL}$ & ESP & Dif & $A L$ & ESP & Dif & $\mathrm{AL}$ & ESP & Dif \\
\hline 1 & 13,2 & 4,1 & 9,1 & 10,7 & 3,6 & 7,1 & 6,9 & 3,6 & 3,3 & 7,6 & 8,2 & 0,5 & 4,9 & 1,4 & 3,6 & 12,8 & 21,8 & 9,0 \\
\hline 2 & 13,6 & 7,7 & 5,9 & 12,1 & 9,1 & 3,0 & 8,0 & 11,8 & 3,8 & 8,8 & 13,6 & 4,8 & 6,5 & 5,0 & 1,5 & 5,4 & 12,3 & 6,9 \\
\hline 3 & 15,4 & 6,8 & 8,6 & 13,8 & 13,2 & 0,6 & 11,9 & 14,1 & 2,2 & 12,0 & 18,6 & 6,6 & 10,8 & 15,0 & 4,2 & 12,4 & 14,5 & 2,2 \\
\hline 4 & 15,8 & 20,0 & 4,2 & 15,5 & 15,9 & 0,4 & 14,2 & 15,5 & 1,2 & 18,4 & 19,5 & 1,2 & 20,2 & 30,9 & 10,7 & 12,1 & 11,8 & 0,3 \\
\hline 5 & 12,6 & 28,6 & 16 & 19,1 & 32,7 & 13,7 & 18,7 & 25,5 & 6,7 & 18,1 & 17,7 & 0,4 & 23,2 & 28,6 & 5,5 & 12,8 & 7,3 & 5,6 \\
\hline 6 & 18,0 & 32,7 & 14,7 & 18,8 & 24,5 & 5,7 & 33,5 & 28,6 & 4,9 & 23,8 & 20,9 & 2,9 & 22,1 & 18,2 & 3,9 & 22,2 & 8,6 & 13,6 \\
\hline$N / C$ & 11,4 & 0,0 & 11,4 & 10,0 & 0,9 & 9,1 & 6,7 & 0,9 & 5,8 & 11,3 & 1,4 & 9,9 & 12,2 & 0,9 & 11,3 & 22,2 & 23,6 & 1,4 \\
\hline
\end{tabular}
Fuente: Elaboración propia.

El ciberespacio se identifica, por tanto, como un posible escenario de inserción laboral. Con relación a esta pregunta, la siguiente tabla muestra una comparativa entre los 16 países estudiados y señala los porcentajes correspondientes únicamente a la opción número 6 (la más valorada). De este modo, se observa que la preferencia de Internet como salida laboral se sitúa alrededor del 15\% en la mayoría de países. Sin embargo, existen tres casos donde la elección de la Red como el escenario preferido para desarrollarse 
profesionalmente presenta datos muy altos. Se trata de Brasil $(39,7 \%)$, Paraguay $(42,9 \%)$ y Cuba $(58,3 \%)$. No es posible, en el marco de este estudio, determinar los porqués de los elevados porcentajes y la posible relación de los mismos con la coyuntura sociopolítica, educativa y económica de cada uno de ellos. En el caso de Cuba, que presenta evidentes problemas en el acceso y conectividad a las tecnologías digitales, resulta llamativo que más de la mitad de los estudiantes hayan señalado, como primera opción, Internet como el medio donde desearían trabajar.

Tabla 5: Comparativo por países del medio preferido por los estudiantes como primera opción

\begin{tabular}{lcccccc}
\hline & Prensa & Radio & Televisión & Fotografía & Internet & Otros \\
\hline Brasil & $10,3 \%$ & $10,3 \%$ & $34,6 \%$ & $18,0 \%$ & $39,7 \%$ & $16,7 \%$ \\
El Salvador & $14,3 \%$ & $11,9 \%$ & $47,6 \%$ & $33,3 \%$ & $14,3 \%$ & $9,5 \%$ \\
Colombia & $26,8 \%$ & $26,8 \%$ & $29,0 \%$ & $27,5 \%$ & $13,0 \%$ & $21,7 \%$ \\
Guatemala & $4,2 \%$ & $15,5 \%$ & $28,2 \%$ & $31,0 \%$ & $28,2 \%$ & $18,3 \%$ \\
\hline España & $32,9 \%$ & $24,8 \%$ & $28,8 \%$ & $20,7 \%$ & $18,0 \%$ & $8,6 \%$ \\
Honduras & $17,8 \%$ & $29,7 \%$ & $57,6 \%$ & $25,4 \%$ & $22,9 \%$ & $11,0 \%$ \\
Argentina & $31,0 \%$ & $29,3 \%$ & $24,1 \%$ & $17,2 \%$ & $25,9 \%$ & $19,0 \%$ \\
México & $9,2 \%$ & $16,9 \%$ & $24,6 \%$ & $20,0 \%$ & $18,5 \%$ & $32,3 \%$ \\
Chile & $34,3 \%$ & $17,9 \%$ & $28,4 \%$ & $20,9 \%$ & $20,9 \%$ & $26,9 \%$ \\
\hline Nicaragua & $16,4 \%$ & $13,7 \%$ & $42,5 \%$ & $21,9 \%$ & $16,4 \%$ & $15,1 \%$ \\
Costa Rica & $80,0 \%$ & $0,0 \%$ & $20,0 \%$ & $20,0 \%$ & $20,0 \%$ & $20,0 \%$ \\
\hline Paraguay & $23,8 \%$ & $33,3 \%$ & $23,8 \%$ & $52,4 \%$ & $42,9 \%$ & $47,6 \%$ \\
Cuba & $12,5 \%$ & $8,3 \%$ & $29,2 \%$ & $20,8 \%$ & $58,3 \%$ & $37,5 \%$ \\
Perú & $5,0 \%$ & $3,3 \%$ & $15,0 \%$ & $15,0 \%$ & $10,0 \%$ & $43,3 \%$ \\
Ecuador & $14,3 \%$ & $0,0 \%$ & $57,1 \%$ & $14,3 \%$ & $28,6 \%$ & $14,3 \%$ \\
\hline R. Dominicana & $12,9 \%$ & $19,4 \%$ & $25,8 \%$ & $16,1 \%$ & $16,1 \%$ & $38,7 \%$ \\
\hline
\end{tabular}

Fuente: Elaboración propia.

Por otro lado, teniendo en cuenta la pregunta anterior, la investigación verificó que prácticamente la totalidad de los estudiantes de comunicación de América Latina y España (99,35\%) pertenecen, al menos, a una red social. A ello, se une que más de un $74 \%$ está presente en más de una de estas plataformas, aspecto que refuerza la importancia que conceden a las plataformas del social media y, al mismo tiempo, denota su solvencia en la creación y gestión de perfiles en las mismas. 
Figura 1: ¿A cuántas redes sociales perteneces?

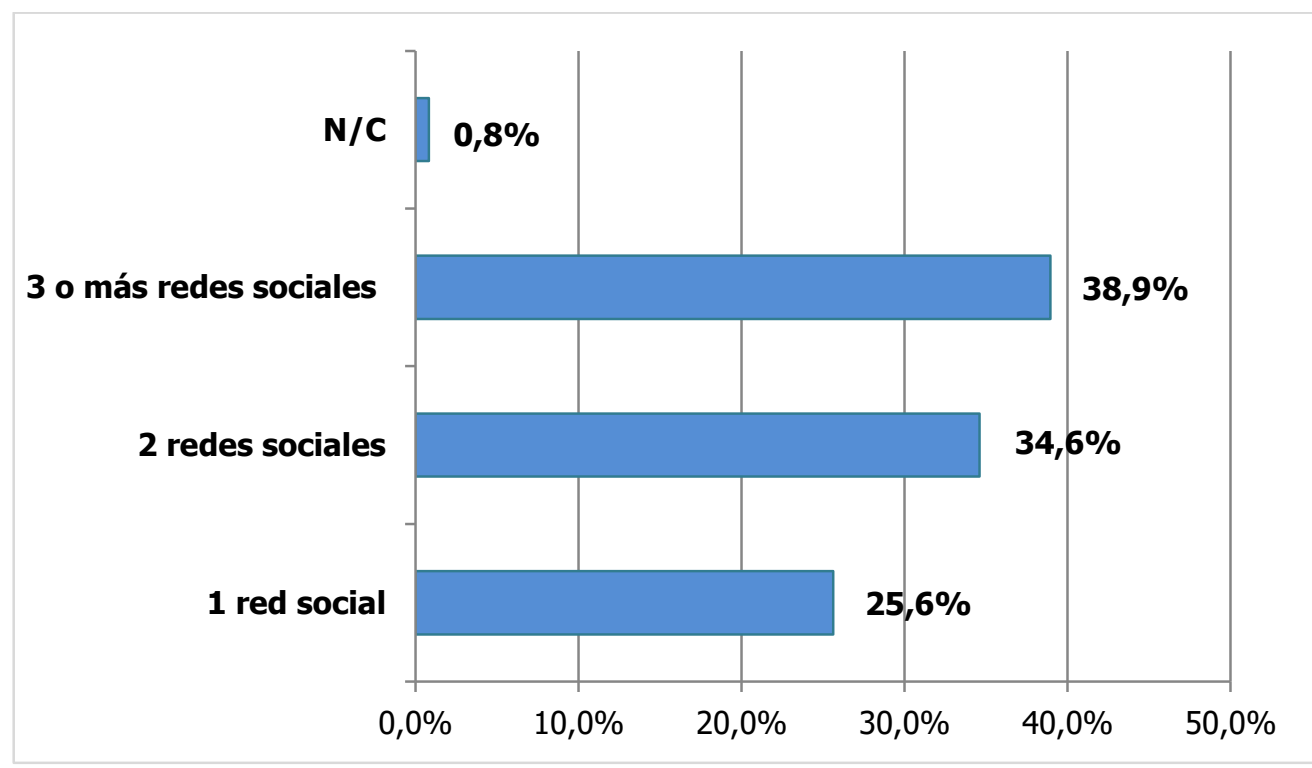

Fuente: Elaboración propia.

El estudio comparativo entre países refuerza el mismo escenario que se mueve entre el 96,9\% de México, el porcentaje más bajo, y el $100 \%$ de la mayoría de países. El matiz vendría dado por el número de redes que poseen. En el conjunto de países se observa una misma tendencia que establece que el grupo más frecuente es el de los estudiantes que poseen perfiles en tres o más redes sociales.

Tabla 6: Comparativo por países sobre el número de redes sociales

\begin{tabular}{lcccc}
\hline & BRASIL & COLOMBIA & ESPANA & ARGENTINA \\
\hline 1 red social & $16,7 \%$ & $25,4 \%$ & $23,4 \%$ & $5,2 \%$ \\
\hline 2 redes sociales & $28,2 \%$ & $58,7 \%$ & $22,1 \%$ & $32,8 \%$ \\
\hline ó más & $55,1 \%$ & $14,5 \%$ & $53,2 \%$ & $62,1 \%$ \\
\hline 1 red social & CHILE & COSTA RICA & CUBA & ECUADOR \\
\hline redes sociales & $22,4 \%$ & $54,5 \%$ & $25 \%$ & $75 \%$ \\
\hline ó más & $38,8 \%$ & $27,3 \%$ & $29,2 \%$ & $14,3 \%$ \\
\hline 1 red social & $38,8 \%$ & $18,2 \%$ & $45,8 \%$ & $10,7 \%$ \\
\hline redes sociales & EL SALVADOR & GUATEMALA & HONDURAS & MEXICO \\
\hline ó más & $50 \%$ & $16,9 \%$ & $52,5 \%$ & $32,3 \%$ \\
& $23,8 \%$ & $45,1 \%$ & $24,6 \%$ & $55,4 \%$ \\
\hline red social & $26,2 \%$ & $38 \%$ & $21,2 \%$ & $9,2 \%$ \\
\hline redes más & NICARAGUA & PARAGUAY & PERU & R. DOMINICANA \\
\hline
\end{tabular}

Fuente: Elaboración propia.

La principal inquietud se centraría en verificar si la utilización de las redes sociales se lleva a cabo con la expectativa de que éstas se consoliden como plataformas que les ofrezcan empleabilidad. Con relación a este aspecto, la investigación revela que "estar informado" (41,9\%) y "pasar el rato" (34,8\%) son los dos grandes usos que los usuarios confieren a las redes sociales. Además, estos resultados generales se 
reproducen al analizar por separado cada uno de los 16 países observados. La búsqueda de trabajo, a partir de las redes sociales, queda relegada a última posición del listado de opciones ofrecidas a los estudiantes (solo un $1 \%$ señala la detección de ofertas de empleo como uno de sus usos en estas plataformas).

Tabla 7: Si perteneces a las redes sociales, ¿para qué la utilizas?

\begin{tabular}{lc}
\hline Tipo de uso & $\mathbf{\%}$ \\
\hline Para informarte. & $41,9 \%$ \\
\hline Para pasar el rato (entretenimiento). & $34,8 \%$ \\
Otros & $6,8 \%$ \\
Para buscar relaciones sociales. & $6,2 \%$ \\
Para encontrar amigos. & $5,8 \%$ \\
Para conocer gente. & $2,1 \%$ \\
N/C & $1,4 \%$ \\
Para buscar trabajo. & $1,0 \%$ \\
\hline
\end{tabular}

Fuente: Elaboración propia.

El dato anterior queda reforzado al interrogar a los estudiantes sobre dos de las principales redes profesionales que existen en la actualidad. Se detecta que más de la mitad de los 1.080 estudiantes consultados conocen LinkedIn $(69,91 \%)$ y un grupo muy reducido $(1,39 \%)$ asegura conocer la red Xing (plataforma on-line de networking que se dedica contactar usuarios o establecer conexiones de tipo profesional). Del total de los alumnos, un grupo muy reducido $(1,85 \%)$ afirma conocer ambas redes; mientras que casi un tercio $(26,85)$ confiesa que desconoce las características y funcionalidades de ellas. Este aspecto permitiría identificar las redes sociales como un "instrumento para encontrar trabajo", pero no permite concluir que los alumnos conciban tales plataformas como espacios donde desempeñarse profesionalmente.

Figura 2: ¿Qué redes sociales profesionales conoces?

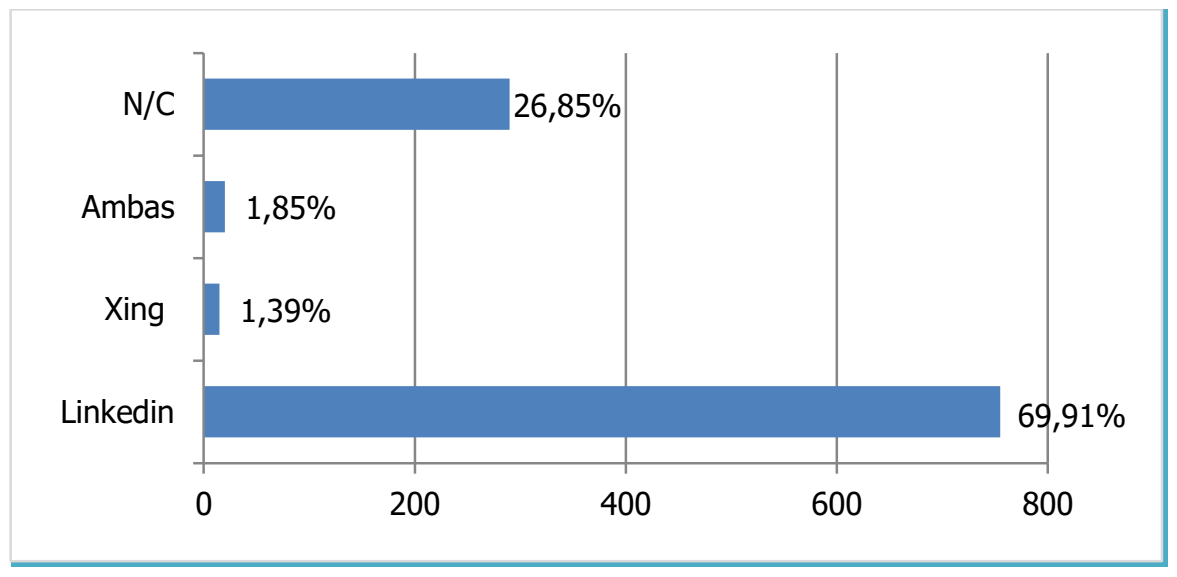

Fuente: Elaboración propia.

A partir de este segundo bloque de datos, se podría establecer que, entre los estudiantes de comunicación, no existe un interés destacado en utilizar o tener presencia en las redes sociales profesionales. De hecho, ninguno de los estudiantes encuestados menciona las redes profesionales como primera opción dentro del listado de redes que usan. De los 186 encuestados que dicen tener un perfil en LinkedIn (17.2\% de la 
muestra), 15 señalan la plataforma profesional como segunda red a la que están conectados, y 171 estudiantes la mencionan como la tercera red. 68 hombres dicen utilizarla al igual que 117 mujeres, en ambos casos se corresponde con el 17\% del total de la respectiva división por género de la muestra. Por el contrario, sí existe una diferencia importante entre los 87 estudiantes que son parte de la red y que pertenecen a universidades privadas, frente a los 99 casos que hacen parte de universidades públicas. Así, mientras LinkedIn tiene una penetración del 21,9\% en los centros de educación superior púbicos, apenas llega al 13,8 en los centros privados. Una misma situación se produce al comparar los espacios geográficos analizados. Mientras en España hay una afiliación que supera el 28\%, en los países de América Latina se estanca en el 14,5\%. Ningún estudiante señaló pertenecer a la red social Xing.

Partiendo de los datos anteriores, obtenidos en la fase diagnóstica de la investigación, se consideró oportuno ahondar en la credibilidad que los estudiantes de América Latina y España conferían a las diferentes fuentes de información que encontraban en las redes sociales. El objetivo era, básicamente, establecer dentro de una plataforma dialógica a qué entidades o emisores de contenidos conferían mayor o menor verosimilitud (mediante una gradación de: "ninguna", "poca", "alguna" y "bastante" credibilidad). Los resultados obtenidos en esta fase del estudio establecen que los estudiantes confieren "bastante" credibilidad a los periodistas $(43,3 \%)$ y a los periódicos $(49,4 \%)$, incluyendo este ítem a cualquier tipo de cibermedio. Las informaciones derivadas de ONG o instituciones sin ánimo de lucro $(42,4 \%)$ ocuparon el tercer lugar de las fuentes con bastante credibilidad. De este bloque de respuestas, se deriva que existe una mayor confianza del estudiantado de Comunicación en las entidades o en las corporaciones cercanas al ecosistema informativo (periódicos, cibermedios, ONG, fundaciones, etc.) y en los periodistas frente a emisores ajenos al ámbito de la comunicación (amigos, personajes famosos, o políticos). Junto a la concepción, señalada anteriormente, de las redes sociales como escenarios de desempeño profesional, se observa una sólida confianza de los futuros profesionales de la comunicación en los contenidos de las redes sociales que han sido emitidos por entidades o profesionales del ámbito del periodismo y la comunicación.

Tabla 8: Credibilidad de las fuentes de información en las redes sociales

\begin{tabular}{|c|c|c|c|c|c|c|}
\hline & $\begin{array}{c}\text { Ninguna } \\
\text { credibilidad }\end{array}$ & $\begin{array}{c}\text { Poca } \\
\text { credibilidad }\end{array}$ & $\begin{array}{c}\text { Alguna } \\
\text { credibilidad }\end{array}$ & $\begin{array}{c}\text { Bastante } \\
\text { credibilidad }\end{array}$ & $\begin{array}{c}\text { Toda la } \\
\text { credibilidad }\end{array}$ & $N / C$ \\
\hline Amigos & $3,2 \%$ & $21,2 \%$ & $37,5 \%$ & $22,8 \%$ & $5,8 \%$ & $9,4 \%$ \\
\hline $\begin{array}{l}\text { ONG/Instituciones } \\
\text { sin ánimo de lucro }\end{array}$ & $2,4 \%$ & $7,0 \%$ & $26,9 \%$ & $42,4 \%$ & $13,9 \%$ & $7,3 \%$ \\
\hline $\begin{array}{l}\text { Personajes } \\
\text { famosos }\end{array}$ & $10,6 \%$ & $36,9 \%$ & $32,9 \%$ & $8,0 \%$ & $2,1 \%$ & $9,6 \%$ \\
\hline $\begin{array}{l}\text { Páginas } \\
\text { gubernamentales }\end{array}$ & $8,0 \%$ & $23,1 \%$ & $28,9 \%$ & $24,4 \%$ & $6,0 \%$ & $9,6 \%$ \\
\hline Políticos & $20,2 \%$ & $38,9 \%$ & $26,0 \%$ & $3,0 \%$ & $0,6 \%$ & $11,3 \%$ \\
\hline Periodistas & $1,0 \%$ & $8,0 \%$ & $31,5 \%$ & $43,3 \%$ & $8,3 \%$ & $7,9 \%$ \\
\hline Periódicos & $1,7 \%$ & $7,0 \%$ & $22,4 \%$ & $49,4 \%$ & $12,7 \%$ & $6,8 \%$ \\
\hline
\end{tabular}

Fuente: Elaboración propia.

Sin embargo, teniendo en consideración los resultados anteriores, resulta contradictorio que solo poco más de la mitad de los encuestados (51,39\%) señale que sigue las informaciones emitidas por, al menos, una red social de un medio de comunicación. Este aspecto denota un aspecto llamativo y hasta cierto punto preocupante. Como se señaló anteriormente, prácticamente la totalidad de los estudiantes de comunicación $(99,35 \%)$ está presente en una red social, pero casi la mitad de ellos $(44,72 \%)$ no sigue los contenidos que 
los medios de comunicación emiten a través de sus redes sociales. Este dato, que cuestiona el uso profesional que hacen actualmente de estas plataformas - y se mantiene similar tanto en los hombres como en las mujeres encuestadas, así como para las zonas geográficas del estudio y para el carácter de las universidades-, plantea la necesidad de estudiar la forma en que los estudiantes de comunicación se informan de la actualidad.

Figura 3: ¿Estás agregado a alguna red social de un medio de comunicación?

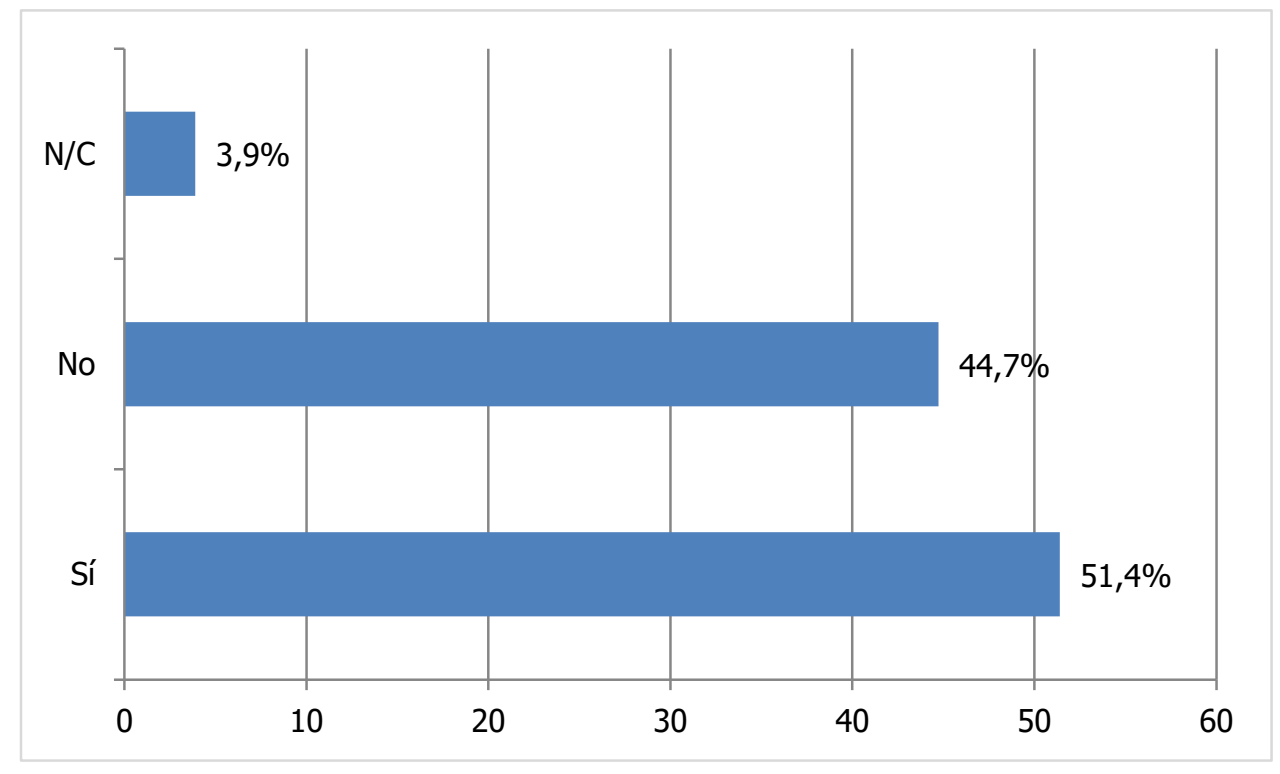

Fuente: Elaboración propia.

Con los resultados anteriores, apareció la inquietud sobre si el escaso uso profesional de las redes sociales por parte de los estudiantes de comunicación encuestados podría justificarse a partir de la proyección en el tiempo que ellos otorgan a las redes sociales. En este sentido, se interrogó a los 1.080 estudiantes sobre este aspecto. De nuevo, los resultados contribuyen a presentar un escenario complejo para el análisis. Una amplia mayoría de los alumnos (79,26\%) considera que las redes sociales no son una "moda pasajera", frente a un grupo más reducido $(17,22 \%)$ que si las califica como plataformas que perderán con el tiempo parte de su importancia. En esta tendencia tampoco hay distinciones significativas entre hombres y mujeres, el carácter de las universidades y las zonas geográficas analizadas.

A ello, se une que, en la última fase de la investigación, casi la totalidad de los encuestados (88,8\%) señaló que una red social es una plataforma donde podría encontrar trabajo o trabajar. Tal y como se detectó en la etapa inicial, se verifica que los estudiantes de comunicación encuestados contemplan las redes sociales como plataformas decisivas en su carrera profesional, aunque no las sitúan entre sus preferencias (que ocupan la televisión y la fotografía). Este aspecto, en último término, vendría a evidenciar que se debería potenciar el trabajo académico en los diferentes planes de estudio dirigido a reflexionar sobre las posibilidades de las redes sociales en el escenario profesional más allá de su rol de ocio o entretenimiento. El conjunto de resultados permite elaborar una aproximación diagnóstica a la concepción que los estudiantes de comunicación de los países de la muestra poseen sobre el potencial de las redes sociales como escenarios para su empleabilidad futura. Los datos evidencian la necesidad de clarificar algunos conceptos entre el 
alumnado, al tiempo que demandan una mayor reflexión sobre el tema durante el proceso formativo con el objetivo de que puedan explotar las posibilidades de inserción laboral que les ofrecen las redes sociales.

\section{Discusión y conclusiones}

La investigación permite señalar que los estudiantes de comunicación de América Latina y España confieren una importancia destacada a las redes sociales, tanto por su presencia en estas plataformas (en muchos casos en más de una) como por la concepción que poseen de las mismas como escenarios sólidos que se proyectan más allá de una mera o simple moda pasajera.

Sin embargo, como señalaban Martínez y Torrado (2011) en su estudio que analizó el uso de las redes sociales como fuente de información, la presente investigación detecta una serie de cuestiones que invitan a la reflexión. Los alumnos señalan, en su mayoría, que utilizan las redes sociales para "estar informados", pero únicamente un grupo muy reducido manifestaba seguir los contenidos de las redes de medios de comunicación. Por tanto, se observa un asunto de interés relativo al tipo de contenidos que los estudiantes y futuros profesionales de la comunicación siguen en sus redes sociales.

Respecto a la concepción y uso de las redes sociales como plataformas de desarrollo profesional, se detectan, de nuevo, algunas contradicciones. Como habían señalado Bulut y Doğan (2017), la investigación detecta que existe una motivación profesional en los estudiantes en el uso de las redes, ya que un $88,8 \%$ concibe las redes sociales como espacios donde encontrar trabajo o trabajar. No obstante, el uso de las mismas para la detección de posibles ofertas profesionales posee una importancia ínfima en el uso que los alumnos hacen de las redes. Este conjunto de datos confronta las conclusiones obtenidas por Baek et al (2011) o Papacharissi y Mendelson (2011) que enfatizaban la importancia para algunos usuarios de utilizar las redes para promocionar un trabajo específico o para publicar avances profesionales.

La investigación invita a una reflexión sobre el tipo de uso y la concepción que los estudiantes de comunicación hacen de las redes. En este sentido, se debería valorar si éstas son vistas como meros "instrumentos" o si son concebidas como "plataformas" autónomas con capacidad de generar ofertas laborales.

Todo ello invita a un debate sobre el tipo de inclusión de las redes sociales a nivel curricular. Pérez Tornero, Tejedor, Simelio y Marín Ochoa (2015) o Salaverría (2016) insisten en la necesidad de adaptar los perfiles profesionales a las exigencias del entorno. Los autores plantean la necesidad de personas cualificadas en la búsqueda, el tratamiento y la difusión de la información en el marco de la sociedad digital, aspecto que abarca a un amplio abanico de perfiles profesionales. Las redes sociales podrían concebirse como escenarios relevantes en el ejercicio profesional de los comunicadores abarcando los diferentes perfiles que van desde los periodistas hasta los gestores, documentalistas, etc.

El impacto de las redes sociales en el escenario informativo digital y la necesidad de adquirir una serie de destrezas para un uso avanzado de las mismas afectan directamente a profesores y alumnos (Roblyer et al, 2010). La practicidad, la inmediatez y el alcance convierten las redes sociales en herramientas periodísticas de gran valor que deben ser, por ello, objeto de una profunda reflexión en el escenario académico (Pérez Tornero, Tejedor, Simelio \& Marín Ochoa, 2015). Este aspecto está estrechamente ligado, como señala Tramullas (2016), a la reformulación de los programas educativos y formativos de las instituciones de 
enseñanza superior que deberían incorporar el conjunto de habilidades y competencias necesarias para un uso avanzado de las redes sociales.

Los resultados del estudio plantean igualmente la necesidad de potenciar las investigaciones que diferencien entre el uso instrumental o técnico de las redes sociales y el manejo crítico y cualitativo de éstas y otras herramientas por parte de los futuros profesionales del periodismo y la comunicación, pero, en general, por el conjunto usuarios (tanto de la generación "milennial" como de las generaciones más jóvenes) que invierten un gran número de horas en el uso de las mismas. A ello se une la necesidad de indagar sobre la relación que existe entre los resultados obtenidos en estudios diagnósticos como éste y la coyuntura política, económica y educativa de cada uno de los países analizados.

\section{Referencias}

Allan, S., \& Thorsen, E. (Eds.). (2009). Citizen Journalism: Global Perspectives. New York: Peter Lang Publishing.

American Press Institute (2016). How Millennials Get News: Inside the habits of America's first digital generation. Arlington: The American Press Institute. Disponible en: https://www.americanpressinstitute.org/publications/reports/survey-research/millennials-news/ Acceso: 22 Jul 2017.

Baek, K., Holton, A., Harp, D., \& Yaschur, C. (2011). "The link that bind: Uncovering novel motivations for linking on Facebook". Computers in Human Behavior, 27 (6), pp. 2243-2248. https://doi.org/10.1016/j.chb.2011.07.003

Bulut, Z. A. \& Doğan, O. (2017). "The ABCD typology: Profile and motivations of Turkish social network sites users". Computers in Human Behavior, 67(2), pp. 73-83. https://doi.org/10.1016/j.chb.2016.10.021

CEPAL (2015). La nueva revolución digital. De la internet del consumo a la internet de la Producción. México: CEPAL. Disponible en: http://repositorio.cepal.org/bitstream/handle/11362/38604/4/S1600780 es.pdf Acceso: 29 Jul 2017.

Diario Gestión (21 de agosto del 2015). "El 37\% de jóvenes cree que las redes sociales son el mejor medio para promover sus productos y servicios". Diario Gestión. Disponible en:

http://gestion.pe/tecnologia/37-jovenes-peruanos-cree-que-redes-sociales-son-mejor-medio-promoversus-productos-y-servicios-2140636 Acceso: 26 Nov 2016.

Francese, P. (2003). "Trend Ticker: Ahead of the Next Wave". Disponible en: http://adage.com/article/american-demographics/trend-ticker-ahead-wave/44208/ Acceso: 29 Jul 2017.

FTI-Ametic (2012). Perfiles profesionales más demandados de la industria de contenidos digitales en España 2012- 2017. Madrid: Fundación Tecnologías de la Información. Disponible en: http://ametic.es/sites/default/files/pafet vii perfiles profesionales cd fti-rooter 1.pdf Acceso: $22 \mathrm{Jul}$ 2017.

Harrison, T., \& Barthel, B. (2009). "Wielding new media in Web 2.0: exploring the history of engagement with the collaborative construction of media products". New Media \& Society, 2 (11), pp. 155-178. https://doi.org/10.1177/1461444808099580 
Martínez, L., \& Torrado, S. (2011). "Uso de las redes sociales como fuente de información de los futuros profesionales de la Comunicación: cilusión o realidad?". En: VIII Foro de Evaluación de la Calidad de la Educación Superior y de la Investigación (FECIES), pp. 359-367.

Matsa, K. E., \& Mitchell, A. (2014). "8 Keys Takeaways about Social Media and News". Pew Research Center - Journalism \& Media, 26 marzo. Disponible en: http://www.journalism.org/2014/03/26/8-keytakeaways-about-social-media-and-news Acceso: 12 Jun 2017.

McCrindle, M. \& Wolfinger, E. (2010). The ABC of XYZ: Understanding the Global Generations. Sydney: UNSW Press.

Ofcom (2015). Public Service Broadcasting in the Internet Age Ofcom's (Third Review of Public Service Broadcasting). Disponible en: https://www.ofcom.org.uk/ data/assets/pdf file/0025/63475/PSBstatement.pdf Acceso: 10 Ene 2017.

Papacharissi, Z., \& Mendelson, A. (2011). "Toward a new(er) sociability: Uses, gratifications and social capital on Facebook". En: Papathanassopoulos, S. (Ed). Media perspectives for the 21st century. Nueva York: Routledge, pp. 212-230.

Pérez Tornero, J. M., Tejedor, S., Simelio, N., \& Marín Ochoa, B. E. (2015). "Estudiantes universitarios antes los retos formativos de las Redes Sociales: el caso de Colombia". Estudios sobre el Mensaje Periodístico, 21 (1), pp. 509-521. http://dx.doi.org/10.5209/rev ESMP.2015.v21.n1.49108

Pew Research Center (2016). State of the News Media 2016. Disponible en: http://assets.pewresearch.org/wp-content/uploads/sites/13/2016/06/30143308/state-of-the-newsmedia-report-2016-final.pdf Acceso: 04 Dic 2016.

Pew Research Center (2014). State of the News Media 2014: Overview. Disponible en: http://www.journalism.org/packages/state-of-the-news-media-2014/ Acceso: 04 Sep 2016.

Roblyer, M., McDaniel, M., Webb, M., Herman, J., \& Witty, J. (2010). "Findings on Facebook in higer education: A comparison of collegue faculty and student uses and perceptions of social networkings sites". The Internet and Higher Education, 3 (13), pp. 134-140. https://doi.org/10.1016/j.i.heduc.2010.03.002

Ruano, L., Congote, E., \& Torres, A. (2016). "Comunicación e interacción por el uso de dispositivos tecnológicos y redes sociales virtuales en estudiantes universitarios". Revista Ibérica de Sistemas e Tecnologias de Información, 19. pp. 15-31. http://dx.doi.org/10.17013/risti.19.15-31

Salaverría, R. (2016). "Redefinir al comunicador". El profesional de la información, 25 (2), pp. 163-167. https://doi.orq/10.3145/epi.2016.mar.02

Tejedor, C., Carniel, R., Giraldo, S. (2018). "Los estudiantes de Comunicación en las redes sociales: Estudio comparativo entre Brasil, Colombia y España". Transinformação, (En preparación).

Telefónica (2014). Telefonica Global Millennial Survey - 2014 Global Results Fact Sheet. Disponible en: http://survey.telefonica.com/es/portfolio/global-results-fact-sheet/ Acceso: 11 Sep 2016

Toffler, A. (1980). The Third Wave. EEUU: Bantam Books.

Tuñez, M., Martínez, Y., \& Abejón, P. (2010). "Nuevos entornos, nuevas demandas, nuevos periodistas". Estudios sobre el Mensaje Periodístico, 16, pp. 79-94. Disponible en: https://revistas.ucm.es/index.php/ESMP/article/viewFile/ESMP1010110079A/11358 Acceso: 31 Ago 2017 
Tramullas, J. (2016). "Hannibal ad portas, o los futuros perfiles profesionales de la información". El profesional de la información, 25(2), pp. 157-162. https://doi.org/10.3145/epi.2016.mar.01

Waycott, J., Bennett, S., Kennedy, G., Dalgarno, B. \& Gray, K. (2010). "Digital divides? Student and Saff perceptions of information and communication technologies". Computers \& Education, 54 (4), pp. 1202-1211. https://doi.org/10.1016/i.compedu.2009.11.006 